\title{
Multi-Label Region Classification and Semantic Linking for Colon Segmentation in CT Colonography
}

\author{
Xiaoyun Yang, Xujiong Ye, Member, IEEE and Greg Slabaugh, Senior Member, IEEE
}

\begin{abstract}
Accurate and automatic colon segmentation from CT images is a crucial step of many clinical applications in CT Colonography, including computer-aided detection (CAD) of colon polyps, 3D virtual flythrough of the colon, and prone/supine registration. However, the existence of adjacent air-filled organs such as the lung, stomach and small intestine, and the collapse of the colon due to poor insufflation, render accurate segmentation of the colon a difficult problem. Extra-colonic components can be categorized into two types based on their $3 \mathrm{D}$ connection to the colon: detached and attached extra-colonic components (DEC and $A E C$ respectively). In this paper, we propose graph inference methods to remove extra-colonic components to achieve a high quality segmentation. We first decompose each 3D air-filled object into a set of 3D regions. A classifier trained with region-level features can be used to identify the colon regions from non-colon regions. After removing obvious DEC, we remove the remaining DEC by modeling the global anatomic structure with an a priori topological constraint and solving a graph inference problem using semantic information provided by a multi-class classifier. Finally, we remove AEC by modeling regions within each 3D object with a hierarchical conditional random field, solved by graph cut. Experimental results demonstrate that our method outperforms a purely discriminative learning method in detecting true colon regions, while decreasing extra-colonic components in challenging clinical data that includes collapsed cases.
\end{abstract}

Index Terms-CT colonography, segmentation, graph inference

\section{INTRODUCTION}

$\mathbf{C}$ OLORECTAL cancer is the second leading cause of cancer related death in western countries [1]. Most colorectal cancers arise from pre-malignant polyps in the colon that develop into cancer over time. The progression from polyp to cancerous lesion takes more than ten years for most patients. Because of this slow growth rate, colon cancer screening [2] is an effective method for polyp detection, and subsequent removal reduces the risk of colorectal cancer by up to 90 percent [3]. In recent years there has been much interest in CT Colonography (CTC) [4], also called "virtual colonoscopy," where a clinical reader screens for colorectal disease using CT images of the cleansed and insufflated colon [5]. Compared to

Xiaoyun Yang is with Hometrack Data Systems Ltd.

Xujiong Ye is with the School of Computer Science, University of Lincoln, LN6 7TS, UK. (email: xye@lincoln.ac.uk).

Greg Slabaugh is with the School of Mathematics, Computer Science, and Engineering, City University London, EC1V OEH, UK. (email: gregory.slabaugh.1@city.ac.uk).

Copyright (c) 2014 IEEE. Personal use of this material is permitted. However, permission to use this material for any other purposes must be obtained from the IEEE by sending an email to pubs-permissions@ieee.org. optical colonoscopy, CTC has advantages that it affords rapid imaging of the entire colon, is less invasive, and has virtually no risk of perforation of the colon. In clinical practice, it is common to scan the patient twice to better identify polyps, once with the patient in supine position, and again in the prone position. This often generates a large quantity of data (typically 800 - 2000 images per patient). Several imaging techniques have been developed to help the clinicians to view and analyze the data more efficiently and effectively. In a "flythrough," a clinician searches for polyps by looking at rendered views of the endoluminal colon surface. In the last decade, many computer aided detection and diagnosis systems [6], [7], [8] have been developed to assist the reader by automatically analyzing the CT data and highlighting potential lesions, boosting reader performance [9], [10]. More recently, advanced prone / supine registration techniques are proposed to better differentiate polyps from pseudopolyps [11], [12], [13]. All of the aforementioned clinical applications rely on automatic and robust colon segmentation.

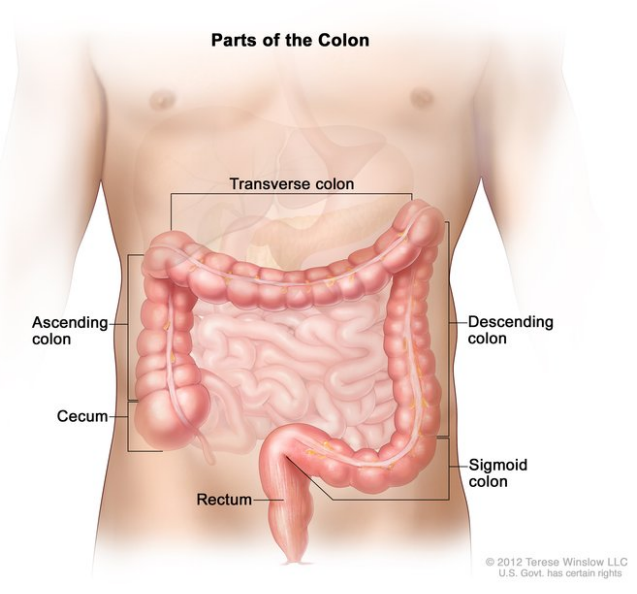

Fig. 1. Illustration of the colon, (license to reproduce the image granted by Terese Winslow).

The colon, located in the abdomen below the stomach and lungs, is separated into five sequential anatomic sections: ascending, transverse, descending, sigmoid and rectum, as shown in Figure 1. When CT scanning is performed, the patient's colon is distended with carbon dioxide (or air), which appears very dark in the CT image. However, for tagging liquid and solid residues in the colon, the patient may have been administered oral contrast agents. In the $\mathrm{CT}$ image, these 
agents appear as bright regions (typically with Hounsfield units $(\mathrm{HU})>300)$. There are two major challenges to robust colon segmentation: first, there are other structures partially or fully filled with air adjacent to the colon, such as lung, small intestine, stomach. These structures may appear $3 D$-connected with the colon as a result of partial volume effect (PVE). Second, ideally the colon is one connected air-filled region as a result of the distension. However, when poorly insufflated, the colon may be collapsed into several disconnected sections. Due to the extra-colonic components, CAD systems may produce out-of-colon false positives [14], which the clinical reader must dismiss and may affect their confidence in the CAD. We classify the extra-colonic components into two categories: detached extra-colonic components (DEC), which are not $3 D$ connected to the colon, and the attached extracolonic components (AEC), which are connected.

\section{A. Related work}

Automatic colon segmentation has been addressed in recent research [14] - [15]. Nappi et al. [16] proposed a knowledgeguided approach to segment the colon in two steps: anatomybased extraction (ABE) and colon-based analysis (CBA). ABE removes extra-colonic components such as bones and lung in an "outside-to-inside" approach; CBA remove digestive extracolonic components such as stomach and small bowels in an "inside-to-outside" approach, by starting a region growing process from a seed placed in the rectum. Multiple seeds are required when presented with partially or completely collapsed colon regions. This method and [16], [17], [18], [14] are more capable of dealing with DEC and not designed for AEC removal. In [19], [20], centerline-based colon segmentation is proposed to remove AEC, but is only valid for well-distended or slightly collapsed colon cases. However, these methods, as well as other published colon segmentation methods require numerous rules and parameter values to be set empirically. Recently [15] is the first to rely on machine learning to solve this problem in a systematic way. This approach first extracts all the $3 D$ air components and applies a binary classifier to classify them into colon components and extra-colonic components. The method then applies a "daisy-chaining" algorithm based on distance to sequentially merge the components between the rectum and cecum. However, due to colon collapses and nearby extra-colonic segments, distance alone can be insufficient to provide robust linking. Furthermore, this method does not address the issue of AEC. Collapsed colons and AEC remain the major barriers to achieve robust segmentation when presented with such difficult cases that are common in clinical practice.

\section{B. Our contributions}

To address these limitations, in this paper we propose a graph inference scheme for colon segmentation using semantic information derived from a multi-class classifier. We first preprocess the data to form a collection of single-connected 3D objects. Each 3D object can be decomposed into and represented by a set of $3 \mathrm{D}$ regions. The obvious non-colon DEC objects are removed if none of its constituent regions is selected by a binary classifier trained from region-level features. A semantic linking strategy is employed to further remove DEC objects. For each remaining object, we identify any AEC regions using a conditional random field (CRF) that incorporates appearance features and spatial dependencies.

The contributions of this paper are three-fold:

1) We introduce a method to detect the colon at a region level. This provides robustness in the presence of collapses or AEC, particularly for salient regions that are more easily discriminated from non-colon regions.

2) We present a semantic linking strategy to guide the colon segmentation using a multi-class classifier to provide the semantic knowledge that indicates to which section of the colon each salient region belongs.

3) We formulate the task of AEC removal in a hierarchical framework as a CRF problem, solved by performing graph cut at each level.

\section{Methodology}

\section{A. Terminology}

Before describing the approach, for clarity, we define some important terms used in this paper. We define a node as a set of connected pixels in 2D. We intend nodes to model the colon on a particular transverse slice forming a 2D image of the 3D volume. However, as there are other adjacent air-filled anatomies near the colon, nodes may exist for extra-colonic image regions as well. Fundamental to this paper is the concept of a region, which is a collection of strongly connected nodes (one can think of a region as a type of supervoxel). The regions group nearby nodes together, and typically nodes in a region will be from the same anatomical section of the colon (e.g., sigmoid colon). Finally, an object is a collection of voxels that are simply connected in 3D. Working directly with objects is problematic because a single object may have AEC or model different anatomical sections of the colon. Therefore, in this paper, we will decompose objects into regions. In terms of set theory, $N \subseteq R \subseteq O$, where $N$ is a $2 \mathrm{D}$ node, $R$ is a $3 \mathrm{D}$ region, and $O$ is a 3D object. Our objective in this paper is to find the set of regions that model the colon, and link them together semantically to form a segmentation.

A schematic diagram showing AEC and DEC, along with objects, regions, nodes, and true positive colon is shown in Figure 2. In the figure, each object enclosed in a red dashed line consists of a number of regions (shown as blue rectangles), while each region has a number of nodes (small yellow circles). The blue dotted dash rectangle indicates true positive colon consisting of three objects (Objects 1, 2, and 4) and Region 1 in Object 3. Therefore, Region 2 in Object 3 is AEC which is attached to the colon; while Object 5 in the figure consists of two DEC regions. The proposed method aims to remove the DEC and AEC while retaining the true positive colon volume.

\section{B. Overview}

Figure 3 provides a high-level overview of the approach. Given a CT image, the method first forms regions, which 


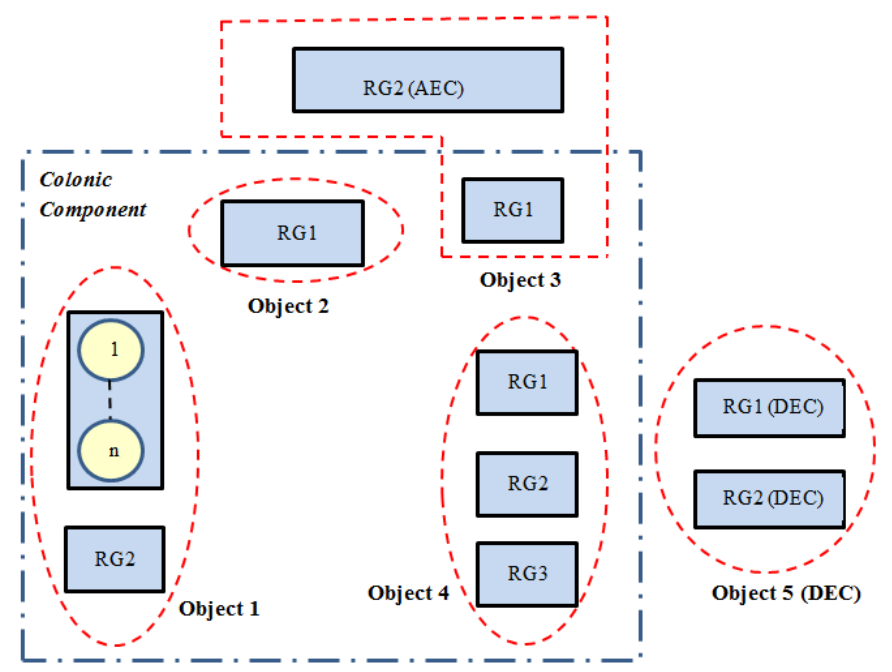

Fig. 2. Schematic diagram illustrating AEC and DEC, along with objects, regions, and nodes.

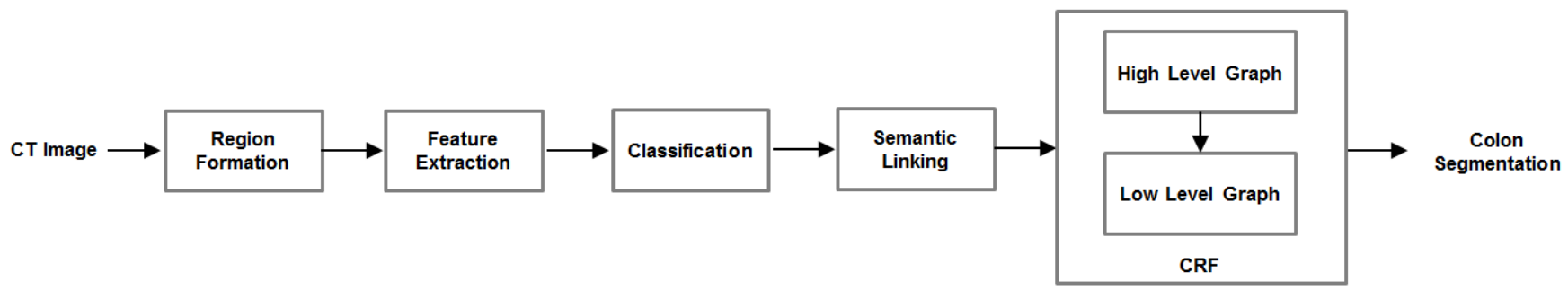

Fig. 3. Overview of the methodology.

consist of strongly connected nodes. Working with regions is advantageous in this problem, since due to partial volume effect and pseudo-enhancement, simple connectivity can group colon with non-colon voxels. Once regions are determined, all subsequent processing is region-based. We extract features that characterize each region's location and shape. The features are used in two classifiers. The first, binary classifier, determines if the region is colon or non-colon. Then, a multi-class classifier categorizes the region into one of six classes, based on the anatomical sections of the colon. A semantic linking strategy uses the anatomic labels to group the regions in the proper order to eliminate remaining DEC, and a final CRF problem is solved to remove any AEC to produce the final segmentation. Below we describe these steps in detail.

\section{Region formation}

1) Image processing: Normally, the colon is insufflated with gas, which appears as a low intensity in the image. However, oral contrast agents (tagging) are often used to opacify any liquid or solid remains in the colon. These contrast agents have a high intensity. Therefore, we first apply pixelwise thresholding on each slice of the input volume based on predefined Hounsfield (HU) values described in Equation 1. Pixels are then labeled as air, high-intensity and background (non-colon), respectively.

$$
f(I)= \begin{cases}\text { air } & \text { if } I<-700 \\ \text { high intensity } & \text { if } I>300 \\ \text { background } & \text { otherwise }\end{cases}
$$

where $I$ is the Hounsfield value of a given pixel. Figure 4 (a) shows a CT slice, and in (b) and (c) the results of detecting the air and liquid regions.

One difficulty to the simple thresholding described above is at the interface between air and liquid. Due to partial volume effect, this "gap area" can have Hounsfield units that are in the background (tissue) class, and can disconnect a set of colonic voxels into disjoint sets. However, this gap area is part of the colon and should be included in the segmentation, and we would prefer these adjacent air and liquid regions merged. We note that the air/liquid interface is small, and flat due to gravity. We use these properties to identify the gap area and merge the colonic air with tagged liquid.

After thresholding, we identify the liquid tagged area on each slice by finding high-intensity pixels neighboring the air. Distance transforms (using the bwdist function in Matlab) are computed from the air pixels and the high intensity pixels in each slice respectively. In the gap between colon air and tagging fluid, the gradient of the respective distance transforms should point oppositely in the vertical direction. We apply a dot product operation with the $y$ (corresponding to gravity) gradients. If the dot product is less than $T_{\text {grady }}$ (e.g., -0.98), we detect that the gradients point oppositely in the vertical direction. Furthermore, the distance value for that pixel from either distance transform should be less than a threshold $T_{\text {GapWidth }}$. The pixels satisfying both conditions are considered as the gap area between colon air and liquid, as shown the dark area in Figure 4 (d). This process is described 


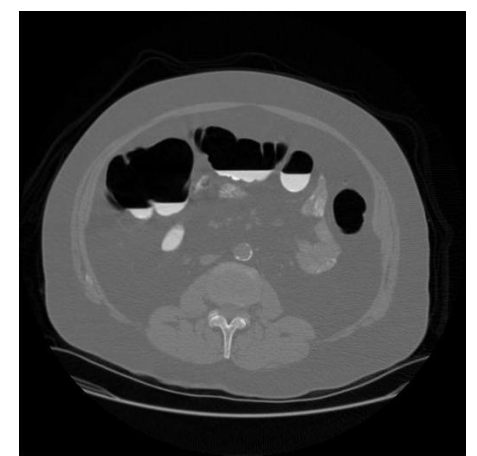

(a) original slice

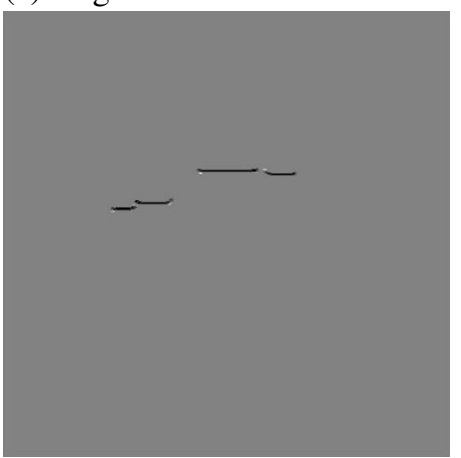

(d)gap area

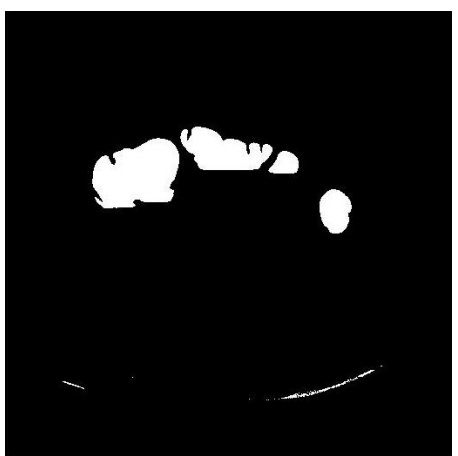

(b) air

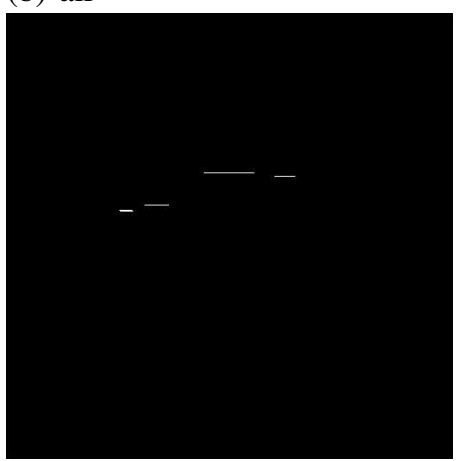

(e) selected gap area

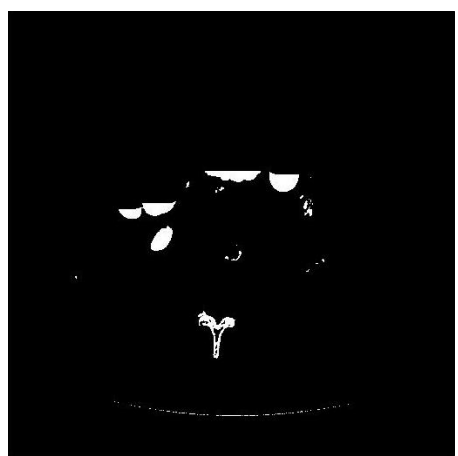

(c) high intensity pixels, $>300$

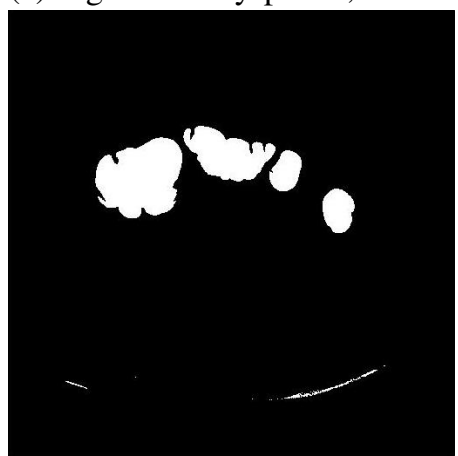

(f) merged colon air and tagging liquid

Fig. 4. Colon air and liquid merging.

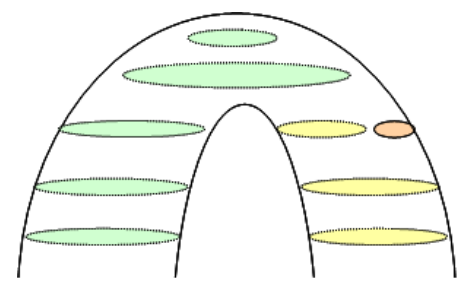

(a)

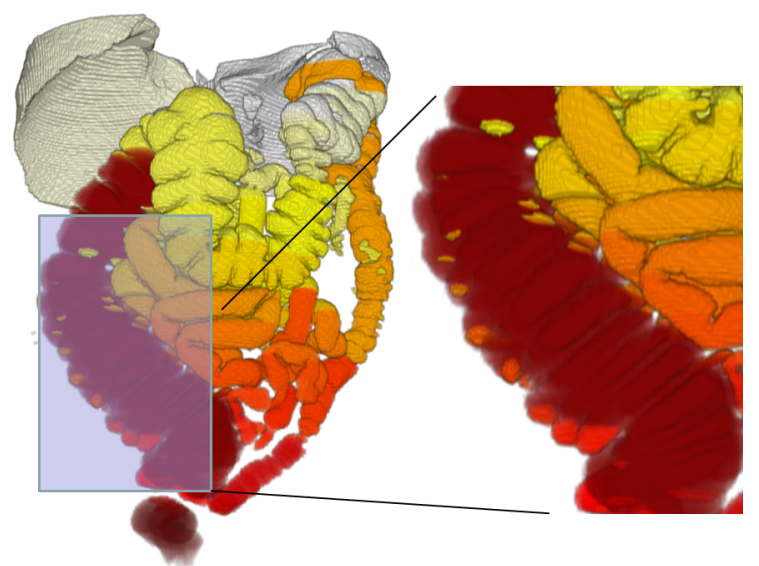

(b)

Fig. 5. In (a), we show a schematic of region formation, consisting of 2D nodes (ellipses) that are grouped into regions (green, orange, yellow). In (b), we show regions identified in a real dataset. A zoom-in is shown for the ascending colon position, where we can see a salient region (coded by dark red) and small fragment regions (coded by light red or yellow).

by Equation 2.

$$
\left\{\begin{aligned}
M_{1}=d t(\text { air })<T_{\text {GapWidth }} \\
M_{2}=d t(\text { high intensity })<T_{\text {GapWidth }} \\
M_{3}=\operatorname{grad}(d t(\text { air })) * \\
\quad \quad g^{*}(d t(\text { high intensity })) \\
M_{4}=\left(M_{3}<T_{\text {grady }}\right) \cdot *\left(M_{1}\right) \cdot *\left(M_{2}\right)
\end{aligned}\right.
$$

where $d t($.$) represents the distance transform operation,$ $\operatorname{grad}_{y}($.$) represents the y$ component of the gradient. For robustness, the area is further filtered by features (area, eccentricity, and the flatness), producing the final gap area illustrated in Figure 4e. The gap area is accepted if area $>3$ pixels, eccentricity $>0.9$, flatness $<0.45)$ (flatness $=$

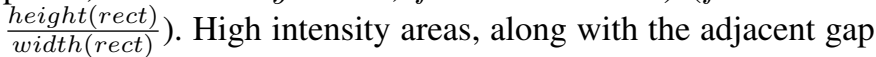
areas are considered as tagged liquid and merged with the colon air using morphological operations.

2) Grouping: We form $3 D$ regions by checking the strength of the connection between constituent $2 D$ nodes on transverse slices; a depiction is shown in Figure 5(a). A node $n_{i}$ in slice $k$ may overlap a node $n_{j}$ in its neighboring slice $k+1$ (or $k-1) . E\left(n_{i}, n_{j}\right)$ represents the Jaccard index between node 
$n_{i}$ and $n_{j}$ and is defined as

$$
E\left(n_{i}, n_{j}\right)=\frac{\operatorname{area}\left(n_{i}\right) \cap \operatorname{area}\left(n_{j}\right)}{\operatorname{area}\left(n_{i}\right) \cup \operatorname{area}\left(n_{j}\right)} .
$$

We define an adjacency matrix $A\left(n_{i}, n_{j}\right)$ to encode the connectivity between nodes. An element of the adjacency matrix is set to one if:

- $E\left(n_{i}, n_{j}\right) \geq 0.5$. In this case, nodes $n_{i}$ and $n_{j}$ are strongly connected and very likely to be part of the same structure.

- $0<E\left(n_{i}, n_{j}\right)<0.5$ and $n_{i}$ doesn't connect with more than two nodes $n_{j}$ and $n_{k}$. This allows for rapid area changes in the $3 \mathrm{D}$ segment when there is no branching connection.

In Figure 5(a) we show different regions within the volume with a different color. Based on the adjacency matrix, the colon is naturally separated into different regions at turning points (such as near the hepatic/splenic flexures) and branching points when connected with other structures. As we will show, this enables the separation of the colon from AEC. However, due to the complex shape of the colon, small fragment regions are often generated (illustrated in Figure 5(b)). The features from these small regions are not discriminative and are not useful for the task of classification.

The $i$ th $3 D$ connected set of voxels forms an object, denoted by $v_{i}$. Using the method described above, each individual object $v_{i}$ can be decomposed into a set of regions $\left(v_{i 1}, v_{i 2}, \ldots, v_{i p}\right)$ that we use in subsequent sections of this paper.

\section{Feature Extraction}

A colon object usually consists of a number of $3 D$ salient colon regions, and non-salient colon regions that are mainly fragments. The salient colon regions have a different geometric appearance and spatial distribution than the non-colon or nonsalient colon regions. To differentiate colon regions (particularly salient ones) from non-colon regions, we developed a set of 114 features listed in Table I (this includes some features from [15]). The features are grouped into three categories. Category 1 are $3 D$ features, computed over the entire $3 D$ region. This includes the $3 D$ enclosing box (represented by six values) and its normalized position (another six values) relative to the bounding box formed from all candidate regions in the volume. Also included is the region's length, approximated by $\frac{\text { area }^{2}}{4 \times \pi \times \text { volume }}$, and the radius, approximated by $\frac{2 \times \text { volume }}{\text { area }}$, where area is the side surface area and volume is the volume of the $3 D$ region.

Category 2 looks at the distribution of the $3 D$ region's features across its constituent $2 D$ nodes on transverse slices. More specifically, we compute the maximum, minimum, mean and median of each measure, specifically area, eccentricity, perimeter, solidity (true area divided by convex area), equivalent diameter, and Euler number of each node.

Finally, category 3 features are based on the holes in each node, as well as concavities along its boundary. A given region may contain a number of nodes; and each node will consist of a set of pixels with a boundary. Holes are found using inverting the node and counting the number of connected components. Intuitively, the number of holes will be low in the colon, but high for non-colon anatomy like the lungs, which contain a large number of a vessels that appear as holes in the original (uninverted) node. Concavities are based on the critical points technique described in Section 4.1 of [14], which analyzes the curvature of points along the boundary of a node to determine a concave area. For both a region's holes and concavities, a set of 9 statistics (number, as well as sum, maximum, minimum, mean, median, variance, entropy, mode of the area forming the hole or concavity) are computed for each transverse slice. Then, a set of 4 summary statistics (maximum, minimum, mean, and median) are computed across all the slices in the region, forming a set of $9 \times 4$ features; one set of 36 features for holes, and another for concavities.

\section{E. Probabilistic classification}

Next, we train two classifiers (a binary and a multi-class classifier) to classify 3D regions. The binary classifier is designed to discriminate between colon and non-colon regions, while the multi-class classifier labels a region into one of the six anatomic colon classes to provide semantic information required for linking the regions as discussed in Section III-A.

For the binary classifier, we use a boosting tree introduced by [21], that consists of an ensemble of a serial of trees built in an additive manner. It uses a tree classifier as a weak learner to generate a hypothesis $h_{1}$. The distribution of weights of the training samples is updated by a function of the classification error. A next hypothesis $h_{2}$ is generated by training a weak classifier on the samples randomly drawn but controlled by the distribution of weights from the training data space. The samples misclassified in the previous round can be drawn repeatedly by chance. This process continues iteratively until a target error bound or maximum number of rounds has been reached. The final hypothesis $H$ is formed by linearly combining the set of trees $\left(h_{1}, h_{2}, \ldots h_{t}\right)$ generated at each round with their weighted votes. During testing, after the classifier has been trained, the probability [21] of a region being colon is estimated as

$$
P\left(c_{i p}^{1} \mid v_{i p}, I, \theta\right)=\frac{\exp \left(F\left(c_{i p}^{1}\right)\right)}{\exp \left(F\left(c_{i p}^{0}\right)\right)+\exp \left(F\left(c_{i p}^{1}\right)\right)}
$$

where $I$ is the volume data and $\theta$ is the model generated by training the classifier. $c_{i p}$ is the set of labels for each candidate region $v_{i p} . c_{i p}^{1}$ represents the region corresponding to colon and $c_{i p}^{0}$ represents the region corresponding to noncolon. $F\left(c_{i p}^{1}\right)$ and $F\left(c_{i p}^{0}\right)$ is the classifier prediction value for colon and non-colon respectively.

The regions with high volume appear more salient, with more discriminative features. Also, misclassification of high volume colon regions is highly undesirable. To differentiate these salient regions, we give more weight to the regions with high volume during classifier training, but less weight to small volume regions. The weight factor is defined by the square root of the region's volume, which penalizes misclassification of high volume regions. During testing, the classifier estimates the probability of each region to be colon, and for any object 


\begin{tabular}{|c|c|c|}
\hline Features & Name & Num features \\
\hline Category 1 & $\begin{array}{c}\text { body position, normalized body position, number of } 2 D \text { regions, } \\
\text { volume, } \frac{\text { volume }}{\text { number(Dregions) }} \text {, surface area, length, radius }\end{array}$ & 18 \\
\hline Category 2 & $\begin{array}{c}\text { area, eccentricity, perimeter, solidity, equivalent diameter, } \\
\text { Euler number }\end{array}$ & $6 \times 4=24$ \\
\hline Category 3 & holes, concavities & $9 \times 4 \times 2=72$ \\
\hline
\end{tabular}

TABLE I

FEATURES OF COLON REGIONS, 114 FEATURES IN TOTAL.

$v_{i}$, if one of its constituent part $v_{i p}$ is assigned to be colon, the whole object $v_{i}$ will be labeled as colon for the subsequent processing. Otherwise, the region is labeled as non-colon and discarded as DEC.

To build a semantic contextual information for the graph inference in the next stage, we employ a multi-class classifier to label the regions into anatomic five sections by their locations: rectum (1), sigmoid (2), descending colon (3), transverse colon (4) and ascending colon (5), class number $m=[0,1, \ldots, 5]$ is arranged in a semantic sequence; the class number is 0 for non-colon. The colon regions are categorized into these classes according to which colon section most of its volume is located. Multi-class LogitBoost [21] directly estimates the multi-class probability as

$$
P\left(l_{i p}^{m} \mid v_{i p}, I, \theta\right)=\frac{\exp \left(F\left(l_{i p}^{m}\right)\right)}{\sum_{l} \exp \left(F\left(l_{i p}^{m}\right)\right)}
$$

where $\theta$ is the model generated by training the classifier, and $l_{i p}^{m}$ represents the region $v_{i p}$ corresponding to colon section $m$. $F\left(l_{i p}^{m}\right)$ is the classifier prediction value of class number $m$ for that candidate region $v_{i p}$. We adopt the same principle to train the multi-class classifier, weighting the misclassification cost of each region with the square root of the region's volume.

For training the binary classifier, we could automatically label each region as colon/non-colon by checking whether the region overlaps with the annotated targeted object. For the multi-class training data, we are only interested in the semantic information of the salient regions. The salient regions (on average 15 per volume) are checked visually to determine which anatomic section of the colon they best represent. The salient regions are the regions with the volume exceeding $1 \%$ of the total volume of the air-filled regions. There are some uncertainties when the regions are crossing two anatomic sections; however, the majority of the regions reflects the distributions of each anatomic section and is suitable to train the multi-class classifier.

The posterior probability of being colon/non-colon estimated by binary classifier and the output of the multi-class classifier are used in subsequent graph inference methods described in the next section.

\section{GRAPH INFERENCE}

In this section, we present a graph scheme with two separate graph inference steps to remove the remaining DEC if existing and AEC respectively.

\section{A. Semantic Linking}

To further remove the DEC, we propose a class-level topology graph to characterize the classes' spatial relationship and use it to guide the linking of each colon regions. A variant of pictorial structures (PS) model [22] is employed to identify the rectum and ascending colon regions. We then solve a minimum path problem between these identified end regions using anatomic constraints induced by the multi-class classifier. This effectively removes the remaining DEC.

1) Rectum and Ascending Colon Identification: We note that the rectum and cecum are distinctive landmarks in the colon and their detection is an important component of related work [15]. In our approach, we do not detect the cecum directly, but rather seek to identify the ascending colon regions, which will naturally include the cecum due to strong connectivity.

PS [22] is a probabilistic model that allows the representation of an object as a collection of regions in an image, which are linked, pairwise, by deformable spring-like connections. Each connection defines the relationship between the two regions it connects. In our approach, the connections correspond to the relative spatial positions between regions. The model can be further formulated into two terms, as shown in Equation 6. The first term describes the probability of a region being the target (rectum or ascending) by its geometric appearance features. The second term describes the spatial configuration of the regions which is encoded through a pair-wise term between regions labelled by the multi-class classifier, as

$$
p(L \mid I, \theta) \propto P(I \mid L, \theta) P(L \mid \theta)
$$

where $L$ represents the region label, and $\theta=(\phi, \psi), \phi$ represents the model derived from individual's appearance features, and $\psi$ describe the interrelationship of objects, and in the PS model can be re-expressed as

$$
\begin{aligned}
p(L \mid I, \theta) & \propto P(I \mid L, \phi) P(L \mid \psi) \\
& \propto P(L \mid I, \phi) P(L \mid \psi)
\end{aligned}
$$

This can also be viewed as a CRF problem. The first term $P(L \mid I, \phi)$ acts as an unary term representing the probability of a label given the region's appearance features. The second term $P(L \mid \psi)$ acts as a pairwise term, to model the relative spatial relationship between regions with different labels. This can be directly approximated estimated by multi-class boosting classifier as described earlier. Equation 7 is approximately equivalent to 


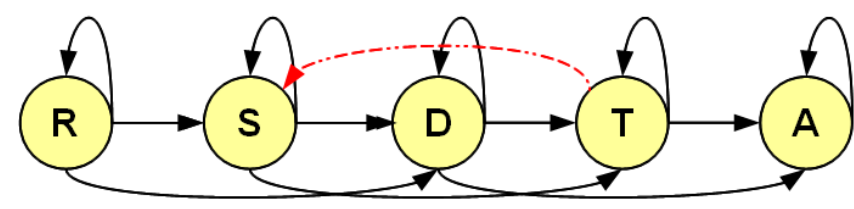

Fig. 6. Class-topology diagram, where (R, S, D, T, A) represent rectum, sigmoid, descending, transverse and ascending sections of the colon respectively. Valid connections are shown in black, and an invalid connection is rendered in red.

$$
p(L \mid I, \theta) \propto \underbrace{\prod p\left(l_{\text {ip }} \mid v_{i p}, I, \phi\right)}_{\text {first term }} \underbrace{\prod p\left(l_{\text {ip }}, l_{j q} \mid \psi\right)}_{\text {second term }}
$$

More specifically, we are looking for two regions, one from the rectum and the other from the ascending colon,

$$
\begin{gathered}
p\left(l_{i p}^{1} \mid v_{i p}, I, \phi\right)=\frac{\exp \left(F\left(l_{i p}^{1}\right)\right)}{\sum_{m} \exp \left(F\left(l_{i p}^{m}\right)\right)} \\
p\left(l_{j q}^{5} \mid v_{j q}, I, \phi\right)=\frac{\exp \left(F\left(l_{j q}^{5}\right)\right)}{\sum_{m} \exp \left(F\left(l_{j q}^{m}\right)\right)} \\
p\left(l_{i p}^{1}, l_{j q}^{5} \mid \psi_{1,5}\right)=N\left(\mathbf{o}\left(v_{j q}\right)-\mathbf{o}\left(v_{i p}\right), \Sigma_{1,5}\right)
\end{gathered}
$$

This equation describes a spatial relationship of two candidates, region $v_{i p}$ being the rectum class $\left(l_{i p}^{1}\right)$, region $v_{j q}$ being the ascending colon class $\left(l_{i p}^{5}\right)$, and their positions $\mathbf{o}\left(v_{i p}\right), \mathbf{o}\left(v_{j q}\right)$ using a multivariate normal distribution. The relative spatial position of the two regions can be represented as a vector $\mathbf{o}\left(v_{j q}\right)-\mathbf{o}\left(v_{i p}\right)$, which is a translation of $\mathbf{o}\left(v_{j q}\right)$ (ascending colon) if using $\mathbf{o}\left(v_{i p}\right)$ as a reference region (rectum). The joint probability for the locations of the regions is based on the deviation between their the observed ones $\left(\mathbf{o}\left(v_{i p}\right), \mathbf{o}\left(v_{j q}\right)\right)$ and their ideal relative values characterized by a covariance matrix $\Sigma_{1,5}$, which is learned from the rectum and ascending colon regions in the training data through maximum likelihood. The covariance matrix $\Sigma_{1,5}$ can be viewed as the stretchiness between two regions. Using $\mathbf{o}\left(v_{i p}\right)$ (rectum) as reference, varying the location of $\mathbf{o}\left(v_{j q}\right)$ (ascending colon) can result in a different cost depending on the covariance matrix $\Sigma_{1,5}$. For example, the ascending colon should be located to the left and above the rectum. A region located to the right (a wrong direction of ascending colon relative to the rectum) will have a low probability and thus be unlikely to be identified as ascending colon.

2) Class-topology graph: The topology of the colon is known a priori in this problem; colon regions should follow a valid, sequential linking from the rectum, sigmoid, descending colon, transverse colon and ascending colon. Ideally, the colon regions are initially linked through the entire colon in the proper anatomic sequence. However, often a colon anatomic section may be broken into a number of disconnected regions. Additionally, the regions may skip the next class in the sequence and instead connect with regions from the class after the next if the colon is collapsed, as shown in Figure 6. Consequently, we define a class-topology graph $G_{c}$, defined by Equation 11

$$
G_{c}=\left[\begin{array}{lllll}
1 & 1 & 1 & t & t \\
t & 1 & 1 & 1 & t \\
t & t & 1 & 1 & 1 \\
t & t & t & 1 & 1 \\
t & t & t & t & 1
\end{array}\right]
$$

where each node represents a class from $m=1,2, \ldots, 5$ sequentially, and use a large value $t$ (representing infinity) to penalize undesired transitions. After we reach one class, the search of regions is limited to regions of the current class, the next class and the class after the next. Backward links or links that skip two classes are unlikely and will be penalized based on Equation 11.

\section{B. Minimum-Cost Path Problem}

After we have the class-topology graph $G_{c}$ and identified two end regions $v_{\text {rectum }}$ and $v_{\text {ascending }}$ of the colon, the problem of identifying the remaining regions representing the colon can be formulated as a minimum-path problem. A graph $G_{p}$ is defined over the set of regions selected by the binary classifier $v_{i p}, v_{j q}, \ldots$ to be colon. A vertex of the graph represents a selected region $v_{i p}$; an adjacency matrix is defined by a weighted distance cost function between two regions, as defined in Equation 12:

$$
W_{p}\left(v_{i p}, v_{j q}\right)= \begin{cases}0 & \text { if } i=j \\ G_{c}\left(v_{i p}, v_{j q}\right) * \Delta\left(v_{i p}, v_{j q}\right) & \text { if } i \neq j\end{cases}
$$

where $\Delta\left(v_{i p}, v_{j q}\right)$ represents a minimum distance between the terminal regions of object $v_{i}$ that contains $v_{i p}$ and the terminal regions of $v_{j}$ that contains $v_{j q}$. Distance between any two regions is based on the Euclidean distance between their bounding box centers. If regions $v_{i p}$ and $v_{j q}$ are from a single simple-connected $3 D$ set $(i=j)$, then the weight in Equation 12 is zero. Otherwise, the two regions $v_{i p}$ and $v_{j q}$ are not from the same $3 D$ object $(i \neq j)$, and their weight is $\Delta$ multiplied by a class-topology matrix $G_{c}$ (defined by Equation 11) which encodes the semantic information of the colon. For example, once the $3 D$ object $\left(v_{i}\right)$ including descending colon regions has been identified $(m=3)$, the $3 D$ object $\left(v_{j}\right)$ identified as transverse colon (at least one region being $m>=3$ ) is expected instead of sigmoid or rectum. To encourage the proper sequence, the pairwise term for out-ofsequence regions is penalized to be very large value $(t=\infty)$. This limits the search range for the subsequent components to the regions from neighboring classes by giving a penalty to the regions from other classes which not admissible based on the class-topology graph. The colon segmentation problem is thus reduced to a minimum path problem between two end regions identified by PS model, and solved by using Floyd-Warshall algorithm [23]. All the $3 D$ objects containing the regions on the resulting path are considered as colon objects and go to subsequent processing. All the other unselected objects are removed as DEC. 


\section{AEC Removal}

After removing DEC in the previous step, we aim to remove AEC in the final stage. For example, Figure 9(a) shows a case of lung attached to a descending colon region, and Figure 9(a, d) illustrate two cases of small intestine connected to the colon (a more commonly occurring phenomenon). Given a 3D object and its constituent regions, we represent the regions of the object on a graph $G_{i}=\left(V_{i}, E_{i}\right)$, where $V_{i}$ is a set of nodes $v_{i 1}, v_{i 2}, \ldots, v_{i k}, E_{i}$ describes the connections between the regions. We seek a labeling $L=1,0$ corresponding to colon or non-colon for each region, and the ones labeled as noncolon are removed as AEC. An assignment of labels to each region is denoted as $\mathbf{y}$. This can be formulated as a CRF [24] problem, where each random variable associates a region with one of the possible labels $L$. This problem can be formulated in terms of energy minimization, as shown in Equation 13:

$$
E(\mathbf{y})=\sum \alpha(\mathbf{y})+\sum \beta(\mathbf{y})
$$

where $\alpha$ is a unary term and $\beta$ is a pair-wise term. The unary term $\alpha$ is represented by the probability of individual object being colon provided their appearance features, which can be estimated by the binary boosting tree classifier described in Equation 4 . The pair-wise term $\beta$ describes the interactions between the regions to enforce a consistency between neighboring regions if they are strongly connected and is described below. Much research has been published to solve this class of optimization problem, and graph cut [25] is proved to be one of the most efficient solutions.

As discussed earlier, we force the 3D object into a set of regions by only checking the strength of the connection between constituent 2D regions on transverse slices. This could lead to undesired small fragments when the 3D shape is complex. The features of these low volume regions are not as distinctive as the features of high volume regions. This may confound the classifier and misclassify these low volume regions of the colon as non-colon.

Therefore, instead of performing the conventional graph inference, we propose a hierarchical graph cut to robustly infer the labels of the constituent regions on the graph: we use the original graph $G_{i}$ that incorporates all the constituent regions of the given object as a low-level graph $G_{i}^{l}$, and from which we can construct a high-level graph $G_{i}^{h}$ that only consists of the subset that are high volume, as shown in Figure 7. CRF can be employed to model the regions and their interactions on each level of the graph. The inference is first started by performing a graph cut on the high-level graph, and the resulting labels are then passed to the same high volume regions on the low level graph as initialization and a low-level graph cut is then performed to infer the labels for all the regions.

On the low-level graph $G_{i}^{l}$, the probability estimation given by the classifier is unreliable for small volume regions, and therefore cannot acted as the unary term. We instead assign 0.5 as their unary term value which gives them a $50 \%$ probability of being colon, and their labels thus depends on the connections and labels of their neighborhood. The pairwise term $\beta(\mathbf{y})$, defined over graph edges, is designed to describe the connection strength (CS) between regions in

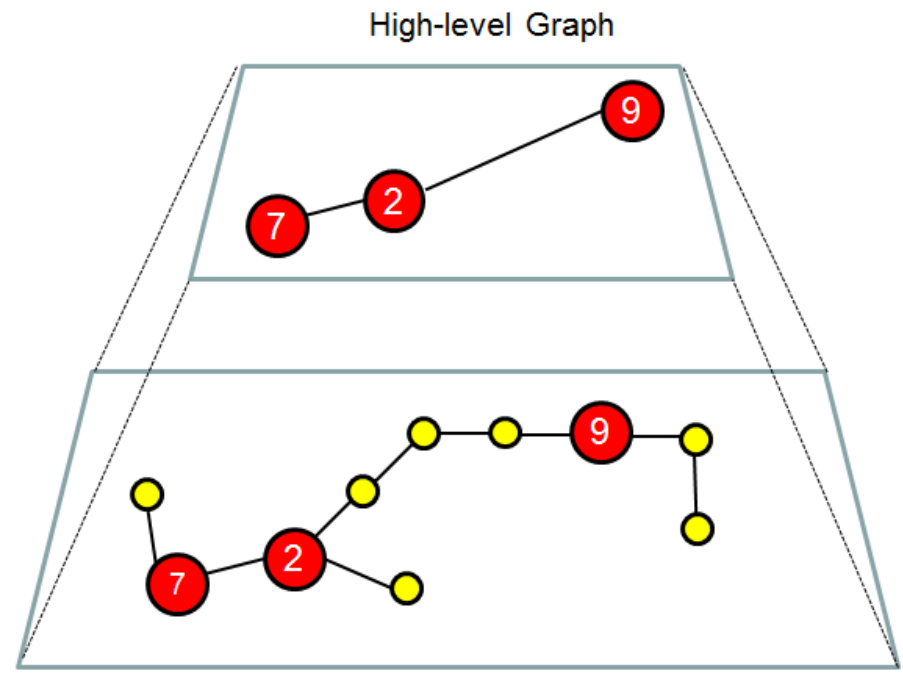

Low-level Graph

Fig. 7. A diagram of a hierarchical graph.

the $3 D$ object. The CS is measured by the Jaccard index of the interfacing $2 D$ regions between the two regions $v_{i}$ and $v_{j}$. We categorize CS into two types: CS between the regions from different structures (e.g., colon and lung) and CS between the regions from the same structure (e.g., both from the colon). The distribution of Jaccard index is modeled as two normal distributions, with mean values 0.35 (between the same structures) and 0.1 (between different structures) learned from a training dataset. We then apply linear discriminant analysis (LDA) [26] to learn the distribution of the CS directly, and the estimated posterior probability from the LDA classifier is used to describe the likelihood that the regions come from the same structure. The value of the pairwise term will be close to 1 if the Jaccard index value between the two regions is large (e.g. above 0.35) and that indicates they are from the same structure, either both are colon or non-colon. Otherwise, the value will be close 0 if the Jaccard index value is low (e.g., 0.01 ) and there will be no constraint to enforce them to have the same labels.

On the high-level graph $G_{i}^{h}$, the features of the high volume regions are distinctive and their probabilities of being colon estimated by the boosting tree classifier can be reliable. We thus use these as the values of unary term. The value of the pair-wise term between any high volume regions in graph $G_{i}^{h}$ is defined to be the minimum of the pair-wise values along the shortest path connecting them at the low level graph $G_{i}^{l}$. In Figure 7, the red nodes are the high volume regions appearing in both graphs. If two regions in high-level graph $G_{h}$ are neighboring in low-level graph $G_{i}^{l}$, such as node 2 and 7 in the figure, the value of pair-wise term between them in $G_{i}^{h}$ is the same value in $G_{i}^{l}$. If the two regions in $G_{i}^{h}$ are not neighboring in $G_{i}^{l}$, such as node 2 and 9 , the value of pair-wise term in $G_{i}^{h}$ is the smallest edge weight on the shortest path between the regions in $G_{i}^{l}$.

In summary, the labeling of the high-level graph is passed to 
the low-level graph to act as unary term for the corresponding regions. Unreliable smaller volume regions in the low-level graph that do not have corresponding regions in the high-level graph are given a unary value of 0.5 , which means that they can be equally likely to be colon or non-colon. A second graph cut performed on the low-level graph gives the final labeling of all the regions on the graph. The regions labeled as non-colon are removed as AEC.

\section{Materials}

We have collected 52 CTC volumes from five different institutions in Europe and the USA using different scanning hardware (GE LightSpeed Plus, GE LightSpeed Ultra, Siemens SOMATOM Plus 4 Volume Zoom). None of the data came from public databases. Patients were prepared using a standard cathartic preparation [27] to eliminate residual waste in the colon before scanning. In addition oral contrast agents were administered so that liquid and solid regions were "tagged," resulting in large (>1000) Hounsfield units. Patients were insufflated with carbon dioxide gas to result in distension of the colon. $\mathrm{kVp}$ ranged from 120 - 140, and exposure ranged from 29 - 500 mAs. Each volume consisted of a set of 512 by 512 image slices. Slice thickness varied from 1.0 to 1.25 $\mathrm{mm}$, with the in-plane resolution from $0.54 \mathrm{~mm}$ to $0.85 \mathrm{~mm}$. The total number of slices for each scan ranged from 294 to 514, with an average of 404. Data was acquired subject to full institutional review board (IRB) approval, and followed ethics protocols to anonymize patient records.

Although standard CTC protocols involve scanning the patient in two orientations (prone and supine), we randomly selected one of these orientations per patient when constructing our dataset (to avoid training one orientation of a patient and testing on the other orientation). As a result, each volume in our data is from a different patient. The 52 volumes were split randomly into 26 training and 26 independent testing datasets, so that no selection bias was present. Annotations made by radiologists were used as ground truth. As described previously, the regions with the volume exceeding $1 \%$ of the total volume of that data after pre-processing were exported into a separate file, and visually checked in which of the five sections they were more likely to be located more likely.

The region formation (Section II-C) was performed using a custom application written in $\mathrm{C}++$ using Visual Studio 2010 (Microsoft, Redmond WA, USA). Subsequent processing was performed using Matlab (Mathworks, Natick MA, USA) version 7.6.

\section{EXPERIMENTAL RESULTS}

We implement an object-level DEC removal method inspired by [15] as our baseline algorithm for comparison. First, we group all the $2 D$ nodes into $3 D$ regions and compute the 114 features for each 3D region to train a boosting tree classifier. $3 D$ regions are selected and output if the chance of being colon estimated by the classifier is greater than of being non-colon. The result is checked against the ground truth. This baseline approach can eliminate DEC but does not address AEC, which is a challenge for data often seen in clinical use.
We defined the following three measures to compare the performance of the methods: FN negative volume and false positive (FP) volume. FN volume measures the volume of colon regions missed by the algorithm. FP volume measures the volume of the extra-colonic components labeled as true colon regions by the algorithm. We additionally compute the Jaccard index of the final colon segmentation with the ground truth. If there are extra non-colon regions remaining in the output, such as lung, stomach or small intestine, the overlap ratio will be low.

In the testing data, 16 out of the 26 volumes have collapses, and 10 out of the 26 volumes have AEC (typically small bowel, lung or stomach). Due to untagged liquid remains, there is one data set with no air in the rectum and therefore no rectum in the segmentation. The PS model correctly identifies the rectum and ascending colon for the other 25 cases, demonstrating the power of the PS model. Figure 8(a-b) illustrates two examples. For the liquid-filled rectum case, it finds the region at the sigmoid section as an alternative to the rectum shown in Figure 8(c). Since the rectal point is shifted in this data set, the model mistakenly picks up a region near the hepatic flexure. However, the selected region and the true ascending colon region are connected in 3D. Measuring the performance at the 3D object level, the PS model has identified the 3D objects containing the rectum or ascending regions with $100 \%$ accuracy respectively.

Table II tabulates the segmentation results on the 26 testing datasets. Among them, 15 volumes are less challenging; our algorithm and the baseline method give the same result. However, the remaining 11 volumes are challenging as most of them have strong presence of both collapses and AEC. False positives generated in step one (binary classification) may be corrected in step two (semantic linking), however, false negatives generated in step one are unrecoverable in step two. Compared to the baseline method that applies a classifier to each 3D object directly, the first step of our method (binary classification) achieves less false negative (FN) volume, but at the expense of more false positive (FP) volume. Such a result is expected at the first step, as the segmentation still includes DEC and AEC, the latter of which is not addressed until the second step of the approach. Compared to the baseline algorithm, the FN volume decreases by $37.8 \%$ but with the FP volume increasing by $17.9 \%$ on average. This shows a better detection rate by introducing some extra-colonic components. However, the overall performance measured by the Jaccard index increases by $8.4 \%$, which demonstrates that the power of the region-level classification, based on the salient regions, is more immune to collapses than a purely discriminative approach.

The second row of the table gives the final result of our graph inference (semantic linking and AEC removal). Compared to the baseline, the FN volume decreases by $9.7 \%$ on average, the FP volume reduces by $63.1 \%$ on average. The improvement in FN reduction is less pronounced that first stage, however, it still outperforms the baseline algorithm by $9.7 \%$ in terms of FN volume reduction. Importantly, the FP volume is hugely decreased by $63.1 \%$ compared to the baseline algorithm. The overall performance increases by 


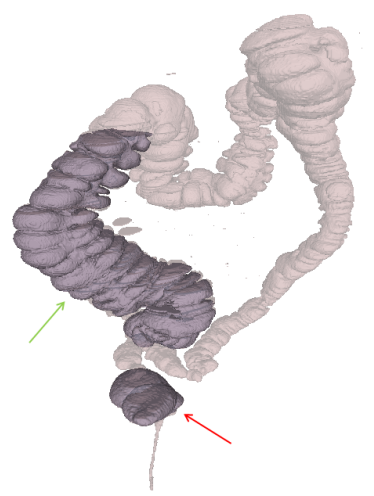

(a)

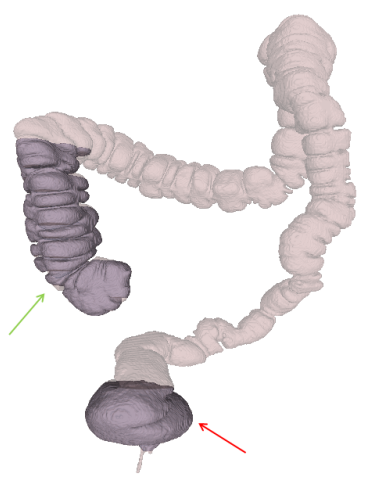

b)

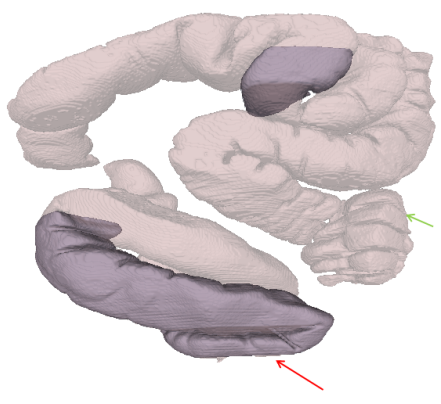

c)

Fig. 8. This figure (a-c) illustrates the results generated by the PS model, where the identified rectum and ascending colon is coded by dark color. The red arrow indicates the place of the rectum and the green arrow indicates the ascending colon. (a)-(b) show two cases with correct detections, (c) the identified ascending region is not at the correct location, but in this data it shares the same $3 \mathrm{D}$ object with the ascending regions and thus no targeted colon is missed in the result.

\begin{tabular}{|c|c|c|c|c|c|c|c|c|c|c|}
\hline \multicolumn{4}{|c|}{ Baseline } & \multirow[b]{2}{*}{ Step } & \multicolumn{3}{|c|}{$\begin{array}{l}\text { Proposed } \\
\text { method }\end{array}$} & \multicolumn{3}{|c|}{ Improvement } \\
\hline & $\begin{array}{c}\text { Jaccard } \\
\text { index }\end{array}$ & FP vol & FN vol & & $\begin{array}{c}\text { Jaccard } \\
\text { index }\end{array}$ & FP vol & FN vol & $\begin{array}{c}\text { Jaccard } \\
\text { index }\end{array}$ & FP vol & FN vol \\
\hline \multirow{2}{*}{$\begin{array}{l}\text { All data (26 } \\
\text { volumes) }\end{array}$} & \multirow{2}{*}{$83.50 \%$} & \multirow{2}{*}{245.3} & \multirow{2}{*}{253.3} & $\begin{array}{l}\text { Step 1: Binary } \\
\text { classifier }\end{array}$ & $80.23 \%$ & 521.7 & 180.7 & $-3.90 \%$ & $-112.67 \%$ & $28.63 \%$ \\
\hline & & & & $\begin{array}{c}\text { Step 2: Graph } \\
\text { inference }\end{array}$ & $87.40 \%$ & 125.9 & 234.5 & $4.60 \%$ & $48.67 \%$ & $7.40 \%$ \\
\hline \multirow{2}{*}{$\begin{array}{c}\text { Challenging } \\
\text { data (11 } \\
\text { volumes) }\end{array}$} & \multirow{2}{*}{$70.20 \%$} & \multirow{2}{*}{447.0} & \multirow{2}{*}{453.6} & $\begin{array}{c}\text { Step 1: Binary } \\
\text { classifier }\end{array}$ & $76.20 \%$ & 527.0 & 282.2 & $8.40 \%$ & $-17.88 \%$ & $37.78 \%$ \\
\hline & & & & $\begin{array}{l}\text { Step 2: Graph } \\
\text { inference }\end{array}$ & $79.30 \%$ & 164.8 & 409.3 & $12.90 \%$ & $63.13 \%$ & $9.77 \%$ \\
\hline
\end{tabular}

TABLE II

RESULTS COMPARED TO THE BASELINE ALGORITHM. WHEN USING THE PROPOSED METHOD ON THE CHALLENGING DATA, THE JACCARD INDEX IMPROVES $12.9 \%$, THE FN VOLUME REDUCES BY 9.7\% AND THE FP VOLUME REDUCES BY 63.1\% ON AVERAGE COMPARED WITH THE BASELINE APPROACH. VOLUMES MEASURED IN $\mathrm{CM}^{3}$, AND IMPROVEMENT IS MEASURED RELATIVE TO THE BASELINE METHOD.

$12.9 \%$ in terms of Jaccard index, outperforming the baseline and the first stage of our method. Quantitatively, these results demonstrate a notable improvement with proposed technique.

Figure 9 shows colon segmentations comparing our proposed method against the baseline. In the examples, although the baseline algorithm has provided good performance, in some data, especially, when the lung, stomach and small intestine are connected with large intestine, it fails to remove those extra-colonic components, which our algorithm better handles. However, under-insufflated colons remain a challenge, particularly those with small volume regions where the colon is collapsed into fragments or appear to be very thin in the CT images. Extra-colonic components that remain belong primarily to lung, small bowel or stomach, and are typically smaller misclassified regions. Our method prioritizes the larger volume regions representing the majority of the colon volume, but as a consequence, smaller volume regions may be harder to disambiguate a colon vs non-colon. Nonetheless, our method produces encouraging results, improving the Jaccard index by $12.9 \%$ for the 11 challenging datasets in our data.

The trained classifiers were tested on a computer with
2.40GHz quad core CPU and 8 GB Memory. On average, it takes about 17 seconds to process the whole image (including loading and writing images) to achieve the colon segmentation.

\section{DisCUSSION AND CONCLUSION}

In this paper, we have presented a novel region-level colon segmentation method that introduces graph inference and multi-class semantics. We tackle the two challenges in colon segmentation in a systematic manner: colon collapses and the removal of attached extra-colonic components (AEC).

We decompose each 3D object into a set of 3D regions, and detect the $3 \mathrm{D}$ colon object from the salient regions that have strong discriminative features. The collapse of the colon may occur at various locations in the colon. In addition, the true colon object may be attached to extra-colon components due to PVE. The AEC could generate a mixing effect at the object-level detection, and learning the generated features may confound the classifier and lower its performance. Trained with the features from solely the salient regions, the classifier model pays more attention to the coherent appearance and spatial features for more consistent performance. 


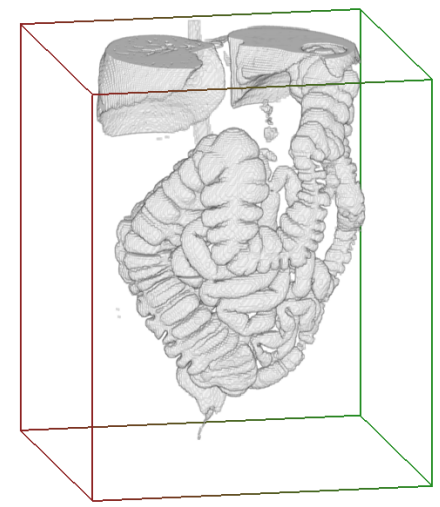

a)

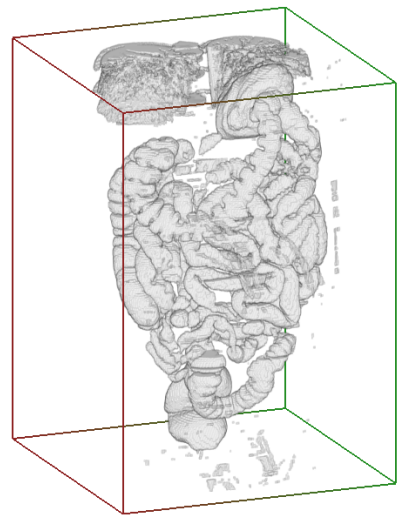

d)

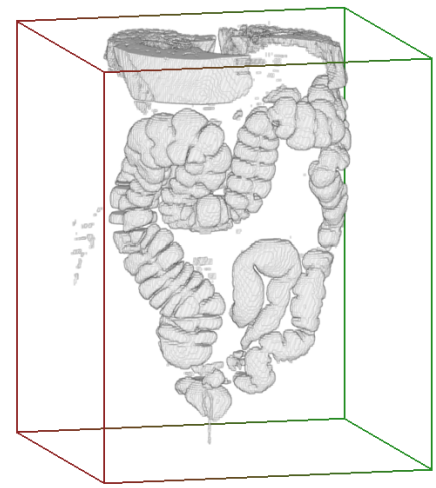

h)

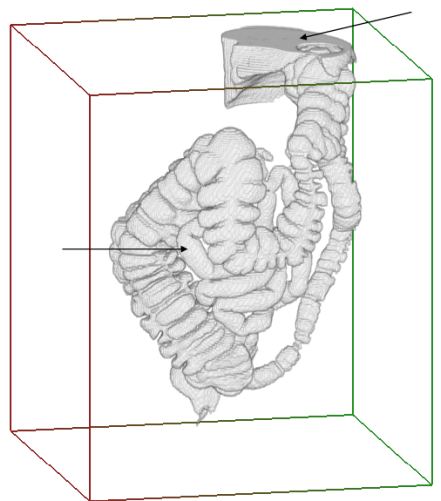

b)

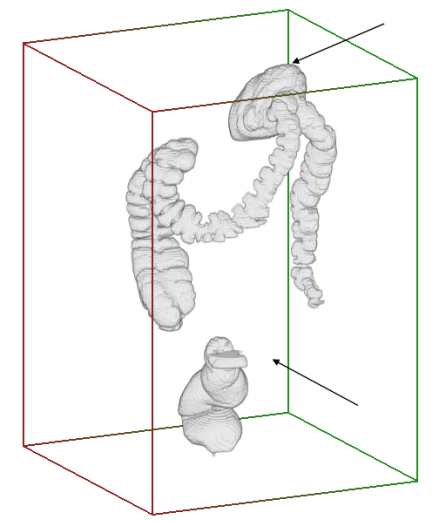

e)

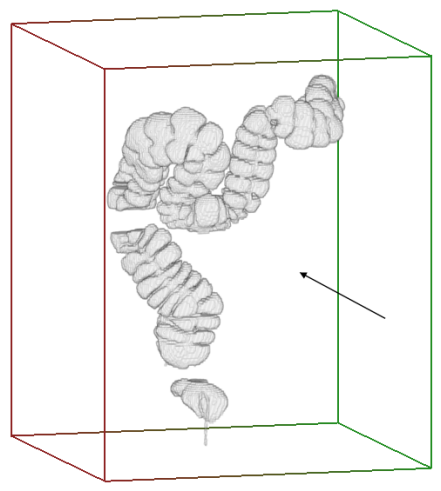

i)

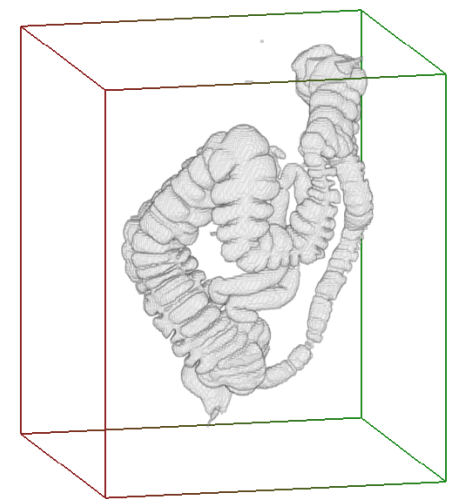

c)

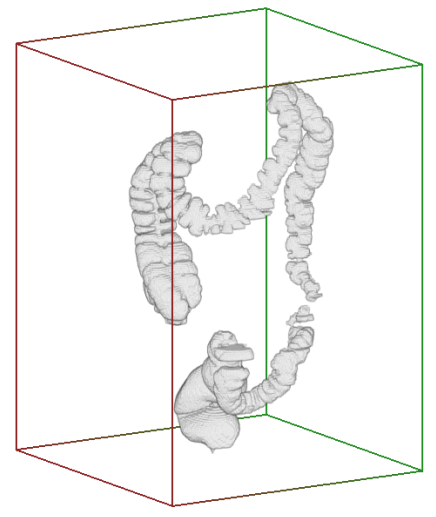

f)

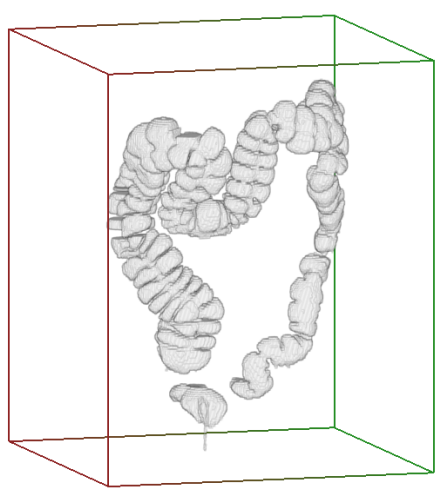

j)

Fig. 9. Performance comparison: the left column ( $\mathrm{a}, \mathrm{d}, \mathrm{h})$ is the input volume, the middle column (b, e, i) is the baseline result, and the last column (c, f, j) is our algorithm result. The figures show that the baseline algorithm failed in removing lung and small intestine portions, and erroneously detects small bowel segments due to collapse, indicated by arrows in the middle column. Our algorithm clearly outperforms the baseline.

When presented collapsed colons, conventional approaches identify the rectum and the ascending colon and sequentially add the colon components to minimize the inter-object distance. However, such methods are difficult to apply robustly to the variations observed in real clinical data. We proposed a semantic linking strategy that sequentially merges the colon components based on their anatomical sequence. To achieve this we employed a variant of the PS model to identify the rectum and ascending colon objects together accurately. Secondly, we build the semantic knowledge with a multi-class labeler from the result of a region-level detection. The inter-object distance guided by the semantic knowledge links the collapsed colon objects sequentially from the rectum to ascending colon. In the removal of AEC, we propose a hierarchical graph cut method to model the dependencies of the regions within each 3D object: for each object, we construct a high-level graph to skip the small volume regions and make the inference between the salient regions. The labeling of the salient regions from the high-level graph is passed to the low-level graph to initiate the inference at the low level graph.

While the results are encouraging, future work could focus on further reduction of both the false negatives and the false 
positives. In particular, false negative regions produced in the first binary classifier step are unrecoverable in the graph inference step that follows. Although our approach includes 114 discriminative features, informed by the literature, to differentiate colon from non-colon, the problem of identifying additional features to improve classification performance remains. In addition, our method assigns more importance to the salient regions of the colon by weighting the classifier training based on the region volume. This provides greater robustness for larger regions, but at the expense of smaller region classification performance. In future work we would like to explore the possibility of training separate classifiers based on region size; however, to do this would require additional datasets beyond those used in this study. Currently, we are most interested in applying the proposed colon segmentation in a CAD pipeline, to evaluate its effect on polyp detection. We expect a considerable improvement, particularly a reduction in false positives produced in non-colon anatomy.

Through our experimental results, we demonstrate that modeling the dependencies between the regions outperforms a pure discriminative learning method at object-level, by improving true detections and reducing extra-components in real, clinical data. These encouraging results can improve the performance of applications that rely on colon segmentation, such as flythrough, $\mathrm{CAD}$, and prone / supine registration.

\section{REFERENCES}

[1] "Cancer facts and figures," American Cancer Society Annual Report, vol. 12, no. 1, pp. 11-12, 2007.

[2] M. E. Zalis, M. A. Barish, J. R. Choi, A. H. Dachmann, H. M. Fenlon, J. T. Ferrucci, S. N. Glick, A. Laghi, M. Macari, E. G. McFarland, M. M. Morrin, P. J. Pickhardt, J. Soto, and J. Yee, "Ct colonography report and data system: A consensus proposal," Radiology, vol. 236, pp. $3-9,2005$.

[3] S. Winawer, "The achievements, impact, and future of the national polyp study," Gastrointestinal Endoscopy, vol. 64, no. 6, pp. 975-978, 2006.

[4] D. J. Vining, Y. Ge, D. K. Ahn, and D. R. Stelts, "Virtual colonscopy with computer-assisted polyp detection," in Computer-Aided Diagnosis in Medical Imaging, K. Doi, H. MacMahon, M. Giger, and K. R. Hoffman, Eds. Elsevier Science, 1999, pp. 445-452.

[5] C. D. Johnson and A. H. Dachman, "Ct colonography: The next colon screening examination?" Radiology, vol. 216, no. 2, pp. 311-319, 2000.

[6] R. M. Summers, C. D. Johnson, L. M. Pusanik, J. D. Malley, and et al., "Automated polyp detection at ct colonography: Feasibility assessment in a human population," Radiology, vol. 219, no. 1, pp. 284-290, 2000.

[7] H. Yoshida and J. Nappi, "Three-dimensional computer-aided diagnosis scheme for detection of colonic polyps," IEEE Transactions on Medical Imaging, vol. 20, no. 12, pp. 1261-1274, 2001.

[8] K. Suzuki, H. Yoshida, J. Nappi, S. G. Armato, and A. H. Dachman, "Mixture of expert 3d massive-training anns for reduction of multiple types of false positives in cad for detection of polyps in ct colonography," Medical Physics, vol. 35, no. 2, pp. 694-703, 2008.

[9] D. Burling, A. Moore, M. Marshall, J. Weldon, C. Gillen, R. Baldwin, K. Smith, P. Pickhardt, L. Honeyfield, and S. Taylor, "Virtual colonoscopy: effect of computer-assisted detection (cad) on radiographer performance," Clinical Radiology, vol. 63, no. 5, pp. 549-556, 2008.

[10] E. Lawrence, P. J. Pickhardt, D. H. Kim, and J. Robbins, "Computeraided detection of colorectal polyps: Diagnostic performance in a large asymptomatic screening population," in International Symposium on Virtual Colonoscopy, 2009.

[11] Z. Lai, J. Hu, C. Liu, V. Taimouri, D. Pai, J. Zhu, J. Xu, and J. Hua, "Intra-patient supine-prone colon registration in ct colonography using shape spectrum," in Medical Image Computing and Computer-Assisted Intervention, 2010, pp. 332-339.

[12] W. Zeng, J. Marino, K. Chaitanya Gurijala, X. Gu, and A. Kaufman, "Supine and prone colon registration using quasi-conformal mapping," IEEE Transactions on Visualization and Computer Graphics, vol. 16, pp. 1348-1357, November 2010.
[13] H. R. Roth, J. R. McClelland, M. Modat, M. J. Cardoso, T. E. Hampshire, M. Hu, S. Ourselin, and D. J. Hawkes, "Registration of the endoluminal surfaces of the colon derived from prone and supine ct colonography," Medical Physics, vol. 38, pp. 1348-1357, May 2011.

[14] G. Slabaugh, X. Yang, X. Ye, R. Boyes, and G. Beddoe, "A robust and fast system for ctc computer-aided detection of colorectal lesions," Algorithms, vol. 3, no. 1, pp. 21-43, 2010. [Online]. Available: http://www.mdpi.com/1999-4893/3/1/21/

[15] L. Lu, M. Wolf, J. Liang, M. Dundar, J. Bi, and M. Salganicoff, "A twolevel approach towards semantic colon segmentation: Removing extracolonic findings," in Medical Image Computing and Computer-Assisted Intervention MICCAI 2009, ser. Lecture Notes in Computer Science. Springer Berlin / Heidelberg, 2009, vol. 5762, pp. 1009-1016.

[16] J. Nappi, A. Dachman, and H. Yoshida, "Automated knowledge-guided segmentation of colonic walls for computerized detection of polyps in ct colonography." J Comput Assist Tomogr, vol. 26, no. 4, pp. 493-504, Jul - Aug 2002.

[17] T. A. Chowdhury, P. F. Whelan, and O. Ghita, "A method for automatic segmentation of collapsed colons at ct colonography," in IICAI, 2005, pp. 3517-3532.

[18] G. Iordanescu, P. Pickhardt, J. Choi, and R. Summers, "Automated seed placement for colon segmentation in computed tomography colonography." Acad Radiol., vol. 12, no. 2, pp. 182-90, Feb 2005.

[19] H. Frimmel, J. Nppi, and H. Yoshida, "Centerline-based colon segmentation for ct colonography." Medical Physics, vol. 32, no. 8, pp. 26652672, 2005.

[20] J. Nappi, H. Frimmel, and H. Yoshida, "Centerline-based colon segmentation for CAD of CT colonography," in SPIE Medical Imaging, vol. 6144 , Mar. 2006, pp. 1767-1774.

[21] J. Friedman, T. Hastie, and R. Tibshirani, "Additive Logistic Regression: a Statistical View of Boosting," The Annals of Statistics, vol. 38, no. 2, 2000.

[22] P. F. Felzenszwalb and D. P. Huttenlocher, "Pictorial structures for object recognition," Int. J. Comput. Vision, vol. 61, no. 1, pp. 55-79, 2005.

[23] T. H. Cormen, C. Stein, R. L. Rivest, and C. E. Leiserson, Introduction to Algorithms, 2nd ed. McGraw-Hill Higher Education, 2001.

[24] H. M. Wallach, "Conditional random fields: An introduction," Tech. Rep., 2004.

[25] Y. Boykov, O. Veksler, and R. Zabih, "Fast approximate energy minimization via graph cuts," IEEE Transactions on Pattern Analysis and Machine Intelligence, vol. 23, pp. 1222-1239, 2001.

[26] T. Hastie, R. Tibshirani, and J. H. Friedman, The elements of statistical learning: data mining, inference, and prediction: with 200 full-color illustrations. New York: Springer-Verlag, 2001.

[27] P. Pickhardt, "Screening CT Colonography: How I Do It," American Journal of Roentgenology, vol. 189, no. 2, 2007. 